\title{
Hyper-Proliferation of Cell Action versus Apoptosis/ Non-Apoptosis in Myc Amplification in Inducing Oncogenesis
}

\author{
Lawrence M Agius* \\ Department of Pathology, University of Malta Medical School, Europe
}

Submission: April 10, 2018; Published: May 09, 2018

"Correspondence Address: Lawrence M Agius, Department of Pathology, Mater Dei Hospital, TAL-QROQQ, University Of Malta Medical School, MSIDA, Malta Europe; Tel: 356-21451752; Email: lawrence.agius@um.edu.mt

\begin{abstract}
The prevention of otherwise enhanced pro-apoptosis is signal targeting for the amplified MYC that induces powerful proliferation-prone dysjunction between contrasting apoptosis and non-apoptosis as evident especially in the context of metabolic over activity and switching to aerobic glycolysis of the Warburg phenomenon. It is the copy-number variability of amplified MYC that induces modulated non-apoptosis that paradoxically is enhanced within contexts of increased protein synthesis and of hyper-proliferation of cells. Additionally, there evolves the constitutive dimensions for transformation that opts for non-apoptosis of highly proliferative cells and of stem cells that are prevented to undergo terminal differentiation.
\end{abstract}

\section{Introduction}

MYC constitutes an integrated panoramic reprogramming agent that extensively controls the range of cell growth factors, metabolic activities and also apoptosis induction. It is widely activated in numerous cancer types in terms especially of amplification of MYC that is expressed as copy-number variation of the transcription factor. Micro-RNAs exert oncogenic progression of various human malignancies and enhance cell proliferation-associated protein expression, cyclin D1 and c-myc [1].

Determined identification allows for the realization of affected expression of hundreds of genes within the nuclear compartment and permits integral response downstream of the MYC pathways. Identification of oncogenic drivers could guide targeted therapy of neoplasms [2]. Heat shock protein a8, clusterin, and N-myc downstream-regulated gene 2 are involved in abnormal cell division, proliferation and carcinogenesis of liver cells during hepatocarcinogenesis [3]. Indeed, apoptosis is induced particularly within a context of growth-stimulation of cells throughout the body.

Such contrast effects allows identification of MYC as constitutively active in practically all of the cells within the body during development. Also, MYC allows a proliferation rather than differentiation of stem cells and also of hematopoietic progenitor cells. Sirtuin 1 is a member of the histone deacetylase family and conceptually there is a distinct similarity between tumorigenesis and cellular reprogramming with improved efficiency in the induced maintenance of the pluripotent state [4].

\section{Contextual Re-Constitution}

Within such constitutive context, the essential amplification, or more rarely translocation of the MYC gene, in oncogenesis portrays the evolutionary conservation of the oncogenic attributes of the MYC pathways. A potential molecular mechanism in which the PIM 1 serine/threonine kinase family member mediates crosstalk between signaling pathways includes different Smad proteins and c-Myc which target downstream transcription factors to trigger EMT [5]. In such terms, permissive growth enhancement and proliferation of affected cells promotes oncogenesis as related to ribosomal biogenesis and of increased protein synthesis. A range of effects of MYC targets the nucleolus in ribosomal biogenesis and also enhanced open chromatin architecture of the nucleus with increased susceptibility to transcription dynamics.

HOTAIR (HOX transcript antisense RNA) mediates an oncogenic action of c-Myc that is essential for breast carcinogenesis and is involved in self-renewal and proliferation [6].

\section{Loss of Integrating Functionality}

Integrative dysfunction of amplified MYC contrasts cell proliferation and growth with an apoptosis-inducing series of protein-protein interactivities within contextual metabolic 


\section{Cancer Therapy \& Oncology International Journal}

hyperactivities of protein synthesis and glucose utilization. MUC13 contributes to rewiring of glucose metabolism in pancreatic cancer and up-regulates the expression of Glut-1, c-Myc and Bcl-2 that are implicated in glucose metabolism [7]. Apoptosis is referable dynamics in terms of the mitochondrial membrane with an enhanced pro-apoptosis that is subject to entry into the programmed cell death mechanistic pathways. P-53 -regulated antioxidant signals are mediators of c-Myc oncogenic functions in viral and non-viral lymphoid tumors and also induce apoptosis as an intrinsic barrier against neoplastic disorders [8]. Both caspase-dependent and caspase-independent pathways in apoptosis induction are modulated by MYC and these changes contrast with the also prevalent proliferation of the affected cells.

A diverted series of induced pathway activities are constitutive components of the developmental programs of cells in general and these are highly conserved during evolution. Only through a controlled interplay between metabolism, extracellular stimuli, and intracellular signaling can successful humour responses be mounted and alteration of this balance can promote oncogenesis, as in B cells, including the involvement of MYC and hypoxia-inducible factor 1-alpha [9]. MYC is one the most common gene abnormalities in oncogenesis and particularly implicates a loss of integrating dimensions of the MYC gene pathways.

\section{Metabolism}

A strictly contextual conditioning of the metabolically hyperactive cell as induced by MYC is also prone to apoptosis and such scenario is propositionally constituted by an amplification of the MYC gene situated within a desert region of the DNA on chromosome 8. Single nucleotide variability is distinctive among patients that develop cancer and is active downstream in the MYC pathway. In such manner, the individual tumor varies in discriminatory mode in individual patients, and allows for a divergent series of pathway progressiveness. Hyper-proliferation of cells is a major problem in osteosarcoma and MicroRNA-204 is an essential regulator in carcinogenesis, including osteosarcoma [10].

Dynamic realization incorporates apoptosis and antiapoptosis in a presumed equilibrium manner within various cell types in the body, and is constitutive of stem cell biology in terms particularly of cell proliferation. Hence, there emerges system progressiveness that is both indicative and also dysfunctionally reconstituted in oncogenesis. It is further to dys-equilibrium dynamics that pro-apoptosis is context-realization in the oncogenesis-promotion effects of amplified MYC action. Antiinflammatory drug resistance selectively promotes putative cancer stem cells in genetically predisposed colon cancer [11].

\section{Hyper-Proliferation}

It would appear significant to consider the contrasting milieu of proliferating cells as induced by amplified MYC to proportionally implicate a series of both apoptotic and non- apoptotic susceptibilities within the range of incident pathway resolution or non-resolution. It is indeed in terms of such milieu contrasts that oncogenesis emerges within the modulated non-apoptosis that in turn operates to promote genomic abnormalities as cells increase proliferative activity. Maternal embryonic leucine zipper kinase (MELK) is a direct transcription target of MYCN and MYC in neuroblastoma and MYCN increases MELK expression via direct promoter binding [12].

Targeting of cells for oncogenesis is a primal dynamics of induced modulation that generally implicates an imbalance between non-apoptosis, growth and proliferation of cells and metabolic hyperactivity with increased protein synthesis and induced Warburg effect on carbohydrate metabolism. Overactive MYC may cause miRNAs' expression deregulation through transcriptional and post-transcriptional mechanisms and epigenetic alterations involving miRNAs dysregulation [13].

In such terms, overall modulation allows the interplay of stem cells and also the constitutive series of cell types distributed within the body. Besides MYC, other members of the MYC gene family include MYCN and MYCL1 that specifically implicate also neuronal-type cells as in neuroblastomas, and hematopoietic cells or small cell lung carcinoma. c-Myc amplification-induced cell cycle dysregulaion is common in oesophageal squamous cell carcinogenesis and constitutes a therapeutic target by JQ1 [14]. In such terms, therefore, constitutive dimensions of reproduction of new cells allows for anti-apoptosis within proliferating cells as dictated by loss of integrating functionality of the MYC pathways. Micro-RNA activity is also modulated by MYC and allows a permissive conditioning of translational pathways of mRNA within further dimensional attempts at antiapoptosis formulation.

\section{Translocation}

In Burkitt's lymphoma, there is $100 \%$ incidence of translocation of the MYC gene that is entirely preserved in integral structure and thus in conditions of preserved MYC amplification/translocation. Propositional terms of reference include the dimensional series of modulatory effects as wellprojected within systems of proliferation of cells and of proapoptosis; the latter is dysfunctionally unbalanced in terms of dys-equilibtrium dynamics of non-apoptosis. It is to be realized that MYC is implicated also in biogenesis of mitochondria and hence influences such apoptosis/anti-apoptosis dysequilibrium. There is poor correlation of mutational landscapes with phenotypes and this limits our understanding of pathogenesis and metastasis of tumors such as pancreatic ductal adenocarcinoma; different oncogenic gains and dosages evolve along distinct evolutionary pathways with constrains and contingencies that vary along defined routes to drive oncogenesis [15].

\section{Contrasting dys-Equilibrium in Transformation}

Hence, there emerges a constitutive series of modulatory roles for MYC that is especially susceptible to copy-number 
variability of the intact gene or of a less common translocation series of changes that transform affected cells. It is further to the essential constitutional dimensions of an intact MYC gene that there is exposed a modulation of hyper-activities and dysequilibrium dynamics due to a severe loss of integrated action of MYC. Tumor Necrosis Factor induces activation of the PI3K/ AKT pathway that negatively regulates differentiation of acute myelogenous leukaemia cells through the re-regulated c-Myc [16]. It is this dimensional loss of integrated action that induces the amplified MYC gene that transforms cells within contexts of prevention of apoptosis. Realization of induced apoptosis on the one hand and of anti-apoptosis is seen as a biologic transformation of the affected cells in terms that are strictly proportional to non-constitutive copy-number variability of the amplified MYC.

\section{Conclusion}

Dimensional dys-equilibrium allows for highly permissive modulation of the principal cell proliferation programs to enhance a contrasting role in apoptosis/non-apoptosis in affected cells that subsequently transform as a functionality of the copy-number variability of amplified MYC. In such terms, the generic component pathways include a loss of integration of MYC modulatory roles as an essential transcription factor within the nuclear compartment. Involvement derivative is projected as an inherent widespread dimensionality of stem cell involvement that appears especially to enhance proliferative potentiality of the cells. It is further to such considerations that the projected loss of integrative action arises as contrasting non-apoptosis in terms paradoxically of a dys-equilibrium with MYC-induced enhancement of apoptosis in constitutive cells of the body. A contrasting profile dimensionality is indeed the acquisition of dysfunctional attributes of proliferating cells that are unable to undergo apoptosis within contextual enhancement of MYC amplification.

\section{References}

1. Zhou ZX, Zhang ZP, Tao ZZ, Tan TZ (2018) MiR-632 promotes laryngeal carcinoma cell proliferation, migration and invasion through negative regulation of GSK3Beta. Oncol Res.

2. Xing D, Zheng G, Schoolmeester JK, Li Z, Pallavajala A, et al. (2018) Next-generation sequencing reveals recurrent somatic mutations in

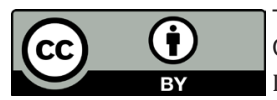

This work is licensed under Creative

Commons Attribution 4.0 License

DOI: $10.19080 /$ CTOIJ.2018.10.555793 small cell neuroendocrine carcinoma of the uterine cervix. Am J Surg Pathol.

3. Sheng X, Huang T, Qin J, Yang L, Sa ZQ, et al. (2018) Identification of the differential expression profiles of serum and tissue proteins during rat hepatocarcinogenesis. Techno Cancer Res Treat 17: 1533034618756785.

4. Ong ALC, Ramasamy TS (2018) Role of Sirtunin1-p53 regulatory axis in aging, cancer and cellular reprogramming ageing. Res Rev 43: 64-80.

5. Zhao B, Liu L, Mao J, Zhang Z, Wang Q et al. (2018) PIM1 mediates epithelial-mesenchymal transition by targeting Smads and c-Myc in the nucleus and potentiates clear-cell renal-cell carcinoma oncogenesis. Cell Death Dis 9(3): 307.

6. Pawlowska E, Szczepanska J, Blasiak J (2017) The long noncoding RNA HOTAIR in breast cancer: does autophagy play a role. Int J Mol Sci 18(11).

7. Kumari S, Khan S, Gupta SC, Kashyap VK, Yaliapu MM, et al. (2018) MUC13 contributes to rewiring of glucose metabolism in pancreatic cancer. Oncogenesis 7(2): 19.

8. Romeo M, Hutchison T, Malu A, White A, Kim J, et al. (2018) The human T-cell leukaemia virus type-1 p3011 protein activates p53 and induces the TIGAR and suppresses oncogene-induced oxidative stress during viral carcinogenesis. Virology 518: 103-115.

9. Franchina DG, Grusdat M, Brenner D (2018) B-cell metabolic remodelling and cancer. Trends Cancer 4(2): 138-150.

10. Zhao R, He H, Zhu Y, Wan J, Li Y, et al. (2017) MiR-204/14-3-3 axis regulates osteosarcoma cell proliferation through SATA3 pathway. Pharmazie 62(10): 593-598.

11. Telang N (2018) Anti-inflammatory drug resistance selects putative cancer stem cells in a cellular model for genetically predisposed colon cancer. Oncol Lett 15(1): 642-648.

12. Guan S, Lu J, Zhao Y, Yu Y, Li H, et al. (2017) MELK is a novel therapeutic target in high-risk neuroblastoma. Oncotarget 9(2): 2591-2602.

13. Barros-Silva D, Costa-Pinheiro P, Duarte H, Sousa EJ, Evangelista AF, et al. (2018) MicroRNA-27A-5p regulation by promoter methylation and MYC singling in prostate carcinogenesis. Cell Death Dis 9(2): 167.

14. Wang J, Liu Z, Wang Z, Wang S, Chen Z, et al. (2018) Targeting c-Myc: JQ1 as a promising option for c-Myc-amplified oesophageal squamous cell carcinoma. Cancer Lett 419: 64-74.

15. Mueller S, Engleitner T, Maresch R, Zukowska M, Lange S, et al. (2018) Evolutionary routes and KRAS dosage define pancreatic cancer phenotypes. Nature 554(6590): 62-68.

16. Li H, Lu N, Yu X, Liu X, Hu P, et al. (2018) Oroxylin A, a natural compound, mitigates the negative effects of TNFalpha-treated acute myelogenous leukaemia cells. Carcinogenesis.

\section{Your next submission with Juniper Publishers} will reach you the below assets

- Quality Editorial service

- Swift Peer Review

- Reprints availability

- E-prints Service

- Manuscript Podcast for convenient understanding

- Global attainment for your research

- Manuscript accessibility in different formats

( Pdf, E-pub, Full Text, Audio)

- Unceasing customer service

Track the below URL for one-step submission https://juniperpublishers.com/online-submission.php 